\title{
A Fifth-fourth Continuous Block Implicit Hybrid Method for the Solution of Third Order Initial Value Problems in Ordinary Differential Equations
}

\author{
Adoghe Lawrence Osa ${ }^{1}$, Omole Ezekiel Olaoluwa ${ }^{2}$ \\ ${ }^{1}$ Department of Mathematics, Ambrose Alli University, Ekpoma, Edo State, Nigeria \\ ${ }^{2}$ Department of Mathematics, Federal University of Technology, Akure, Nigeria
}

Email address:

adolaw@aauekpoma.edu.ng(A. L. Osa), adoghelarry@gma1l.com(A. L. Osa), omolez247@gma1..com(O. E. Olaoluwa)

\section{To cite this article:}

Adoghe Lawrence Osa, Omole Ezekiel Olaoluwa. A Fifth-fourth Continuous Block Implicit Hybrid Method for the Solution of Third Order Initial Value Problems in Ordinary Differential Equations. Applied and Computational Mathematics. Vol. 8, No. 3, 2019 , pp. $50-57$. doi: $10.11648 /$ j.acm.20190803.11

Received: January 30, 2019; Accepted: March 17, 2019; Published: August 12, 2019

\begin{abstract}
In this paper, block method was developed using method of collocation and interpolation of power series as approximate solution to give a system of non linear equations which is solved to give a continuous hybrid linear multistep method. The continuous hybrid linear multistep method is solved for the independent solutions to give a continuous hybrid block method which is then evaluated at some selected grid points to give a discrete block method. The basic properties of the discrete block method were investigated and found to be zero stable, consistent and convergent. The derived scheme was tested on some numerical examples and was found to give better approximation than the existing method.
\end{abstract}

Keywords: Collocation, Interpolation, Approximate Solution, Continuous Block Method, Discrete Block Method, Convergence

\section{Introduction}

This paper considered the approximate methods of solution of the general third order initial value problems of the form

$$
y^{\prime \prime \prime}=f\left(x, y, y^{\prime}, y^{\prime \prime}\right), y(0)=a_{0}, y^{\prime}(0)=b_{0}, y^{\prime \prime}(0)=c_{0}
$$

Where $\mathrm{f}$ is continuous within the interval of integration.

Equation (1) has a wide application in Engineering, Thermodynamics and other real life problems; hence it is important for researchers to study the methods of solving (1).

However, only a limited number of analytical methods are available for solving (1), hence the resort to numerical approximation methods. The well-known conventional method for solving (1) is to reduce it to a system of first order differential equations, Fatunla 1988)

The reduction of such problems of type (1) to systems of first-order equations, leads to serious computational burden as well as wastage in computer time

It has been reported that direct method for solving (1) is more efficient than the method of reduction to system of first order ordinary differential equations [1-18].

Implicit linear multistep methods which have better stability properties than explicit methods have been developed for the solution of (1) above [3-6, 10, 13, 17], among others proposed multi-derivative linear multistep method were implemented in predictor-corrector mode. Although successes were recorded by these methods, the major setback of the predictor-corrector is that apart from the computational burden associated with the method, the predictors are in reducing order of accuracy; hence, the method does not give better approximation.

To cater for these set-backs of the predictor corrector methods, researchers developed block methods. Block method have been developed individually using different approximate solutions (see [1-2, 13, 15-17]). It was found out that block method is more efficient in terms of time of execution, cost effectiveness and accuracy than the predictorcorrector method.

This paper proposes hybrid methods with constant stepsize implemented in block form for the solution of (1). The 
new method preserves the traditional Runge-kutta advantage of being self-starting. The paper is organized as follows: Section two considers the method and the materials for the development of method. Section three considers the analysis of the basic properties of the method which include, zero stability, consistency and convergent. Section four considers numerical examples where the efficiency of the derived method is tested on some numerical examples. Section five considers the discussion of results and the finally chapter six is the conclusion.

\section{Development of Method}

Taylor series expansion of exponential function is adopted as a basis function for the approximation of (1)

$$
Y(x)=\sum_{j=0}^{r+s-1} a_{j} \phi_{j}(x)
$$

Where $\phi_{j}(x)=\frac{x^{j}}{j !}$ and $w_{j}{ }^{\prime} s$ are the coefficients to be determined and a polynomial of degree $r+s-1$. The 5Point hybrid Computational method is constructed by imposing the following conditions on (2).

$$
\begin{gathered}
Y\left(x_{n+j}\right)=y_{n+j}, j=0,1,2, \ldots, r-1 \\
Y^{\prime \prime \prime}\left(x_{n+j}\right)=f_{n+j}, j=0,1,2, \ldots, s-1
\end{gathered}
$$

Putting (1) into (4) gives

$$
f\left(x, y, y^{\prime}, y^{\prime \prime}\right)=\left[\phi_{j}(x)\right]^{\prime \prime}
$$

Here, a step-length of $k=\frac{5}{4}$ with constant step size $h=\frac{1}{4}$. Interpolating (3) at $x=x_{n+j}, j=\frac{1}{4}, \frac{1}{2}, \frac{3}{4}$ and collocating (4) at $x=x_{n+j}, j=0\left(\frac{1}{4}\right) \frac{5}{4}$ gives a system of non-linear equation of the form

$$
Q X=B
$$

Solving (6) for the $a_{j}, j=0(1) 8$ and substituting back into (2) above and after much algebraic simplification yield a method of the form

$$
y(x)=\sum_{j=0}^{k-1} \alpha_{j}(x) y_{n+j}+h^{3}\left(\sum_{j=0}^{k} \beta_{j}(x) f_{n+j}+\beta_{v}(x) f_{n+v}\right)
$$

where $y(x)$ is the numerical solution of the ivp and $v=\frac{1}{4}, \frac{1}{2}, \frac{3}{4}, \frac{5}{4} \cdot \alpha_{j}$ and $\beta_{j}$ are constants and $f_{n+j}=f\left(x_{n+j}, y_{n+j}, y_{n+j}^{\prime}, y_{n+j}^{\prime \prime}\right)$

$$
A^{(0)} Y_{m+1}=\sum_{i=0}^{k} \frac{(j h)^{i}}{i !} e_{i} y_{n}^{(i)}+h^{3-i}\left[d_{i} f\left(y_{n}\right)+b_{i} F\left(Y_{m}\right)\right]
$$

Where

$$
Y_{m+1}=\left[\begin{array}{c}
y_{n+1} \\
y_{n+\frac{1}{2}} \\
y_{n+\frac{1}{4}} \\
y_{n+\frac{3}{4}} \\
y_{n+\frac{5}{4}}
\end{array}\right], f\left(Y_{m}\right)=\left[\begin{array}{c}
f_{n+1} \\
f_{n+\frac{1}{2}} \\
f_{n+\frac{1}{4}}^{4} \\
f_{n+\frac{3}{4}} \\
f_{n+\frac{5}{4}}
\end{array}\right], y_{n}^{(i)}=\left[\begin{array}{c}
y_{n+1}^{(i)} \\
y^{(i)} \\
n+\frac{1}{2} \\
y^{(i)} \\
n+\frac{1}{4} \\
y^{(i)} \\
n+\frac{3}{4} \\
y_{n+\frac{5}{4}}^{(i)}
\end{array}\right], f\left(y_{n}\right)=\left[\begin{array}{c}
f_{n-\frac{3}{4}} \\
f_{n-\frac{1}{2}} \\
f_{n-\frac{1}{4}} \\
f_{n-1} \\
y_{n}
\end{array}\right]
$$

Thus (6) reduces to the following

$$
A^{(0)}=\left[\begin{array}{lllll}
1 & 0 & 0 & 0 & 0 \\
0 & 1 & 0 & 0 & 0 \\
0 & 0 & 1 & 0 & 0 \\
0 & 0 & 0 & 1 & 0 \\
0 & 0 & 0 & 0 & 1
\end{array}\right]
$$

When $i=0$ 
when $i=1$

$$
\begin{aligned}
& e_{0}=\left[\begin{array}{lllll}
0 & 0 & 0 & 0 & 1 \\
0 & 0 & 0 & 0 & 1 \\
0 & 0 & 0 & 0 & 1 \\
0 & 0 & 0 & 0 & 1 \\
0 & 0 & 0 & 0 & 1
\end{array}\right], e_{1}=\left[\begin{array}{ccccc}
0 & 0 & 0 & 0 & \frac{1}{4} \\
0 & 0 & 0 & 0 & \frac{1}{2} \\
0 & 0 & 0 & 0 & \frac{3}{4} \\
0 & 0 & 0 & 0 & 1 \\
0 & 0 & 0 & 0 & \frac{5}{4}
\end{array}\right], e_{2}=\left[\begin{array}{ccccc}
0 & 0 & 0 & 0 & \frac{1}{32} \\
0 & 0 & 0 & 0 & \frac{1}{8} \\
0 & 0 & 0 & 0 & \frac{9}{32} \\
0 & 0 & 0 & 0 & \frac{1}{2} \\
0 & 0 & 0 & 0 & \frac{25}{32}
\end{array}\right], d_{0}=\left[\begin{array}{ccccc}
0 & 0 & 0 & 0 & \frac{3129}{2580480} \\
0 & 0 & 0 & 0 & \frac{317}{40320} \\
0 & 0 & 0 & 0 & \frac{783}{40960} \\
0 & 0 & 0 & 0 & \frac{89}{2520} \\
0 & 0 & 0 & 0 & \frac{-29125}{516096}
\end{array}\right] \\
& b_{0}=\left[\begin{array}{cccccc}
\frac{975}{516096} & \frac{-1931}{12910240} & \frac{173}{184320} & \frac{-1539}{286720} & \frac{139}{2580480} \\
\frac{367}{20160} & \frac{-19}{2016} & \frac{61}{10080} & \frac{-89}{40320} & \frac{1}{2880} \\
\frac{16119}{286720} & \frac{-2187}{143360} & \frac{423}{28672} & \frac{-1539}{286720} & \frac{243}{286720} \\
\frac{73}{630} & \frac{-1}{90} & \frac{11}{315} & \frac{-5}{504} & \frac{1}{630} \\
\frac{101875}{516096} & \frac{625}{258048} & \frac{19375}{258048} & \frac{-625}{73728} & \frac{1375}{516096}
\end{array}\right]
\end{aligned}
$$

$$
e_{1}=\left[\begin{array}{lllll}
0 & 0 & 0 & 0 & 1 \\
0 & 0 & 0 & 0 & 1 \\
0 & 0 & 0 & 0 & 1 \\
0 & 0 & 0 & 0 & 1 \\
0 & 0 & 0 & 0 & 1
\end{array}\right], e_{2}=\left[\begin{array}{lllll}
0 & 0 & 0 & 0 & \frac{1}{4} \\
0 & 0 & 0 & 0 & \frac{1}{2} \\
0 & 0 & 0 & 0 & \frac{3}{4} \\
0 & 0 & 0 & 0 & 1 \\
0 & 0 & 0 & 0 & \frac{5}{4}
\end{array}\right], d_{1}=\left[\begin{array}{cccccc}
0 & 0 & 0 & 0 & \frac{1231}{80640} \\
0 & 0 & 0 & 0 & \frac{71}{2016} \\
0 & 0 & 0 & 0 & \frac{123}{2240} \\
0 & 0 & 0 & 0 & \frac{47}{630} \\
0 & 0 & 0 & 0 & \frac{1525}{16128}
\end{array}\right], b_{1}=\left[\begin{array}{ccccc}
\frac{863}{32256} & \frac{-761}{40320} & \frac{341}{80640} & \frac{94}{80640} & \frac{107}{161280} \\
\frac{34}{315} & \frac{-37}{1008} & \frac{17}{630} & \frac{-101}{10080} & \frac{1}{630} \\
\frac{3501}{17920} & \frac{-9}{2240} & \frac{87}{1792} & \frac{-9}{560} & \frac{9}{3584} \\
\frac{89}{315} & \frac{11}{315} & \frac{38}{315} & \frac{-1}{63} & \frac{1}{315} \\
\frac{11875}{32256} & \frac{625}{8064} & \frac{3125}{16128} & \frac{625}{16128} & \frac{275}{32256}
\end{array}\right]
$$

when $i=2$

$$
e_{2}=\left[\begin{array}{lllll}
0 & 0 & 0 & 0 & 1 \\
0 & 0 & 0 & 0 & 1 \\
0 & 0 & 0 & 0 & 1 \\
0 & 0 & 0 & 0 & 1 \\
0 & 0 & 0 & 0 & 1
\end{array}\right], d_{2}=\left[\begin{array}{ccccc}
0 & 0 & 0 & 0 & \frac{95}{1152} \\
0 & 0 & 0 & 0 & \frac{7}{90} \\
0 & 0 & 0 & 0 & \frac{51}{640} \\
0 & 0 & 0 & 0 & \frac{7}{90} \\
0 & 0 & 0 & 0 & \frac{95}{1152}
\end{array}\right], b_{2}=\left[\begin{array}{ccccc}
\frac{1427}{5760} & \frac{-133}{960} & \frac{241}{2880} & \frac{-173}{5760} & \frac{35}{640} \\
\frac{43}{120} & \frac{7}{180} & \frac{7}{180} & \frac{-1}{60} & \frac{1}{360} \\
\frac{219}{640} & \frac{57}{320} & \frac{57}{320} & \frac{-21}{640} & \frac{3}{640} \\
\frac{16}{45} & \frac{2}{15} & \frac{16}{45} & \frac{7}{90} & 0 \\
\frac{125}{384} & \frac{125}{576} & \frac{125}{576} & \frac{125}{384} & \frac{95}{1152}
\end{array}\right]
$$

\section{Basic Properties of the Methods}

\subsection{Order and Error Constant of the Main Method}

Let the linear Operator defined on the method be $[y(x) ; h]$, where

$$
\Delta[y(x) ; h]=A^{(o)} Y_{m}^{(i)}-\sum_{i=0}^{k} \frac{j h}{i} y_{n}^{\prime}-h^{(3-i)}\left[d_{i} f\left(y_{m}\right)+b_{i} F\left(y_{m}\right)\right]
$$


Expanding the form $Y_{m}$ and $F\left(y_{m}\right)$ in Taylor Series and comparing coefficients in powers of h, we obtained

$$
. \Delta[y(x) ; h]=C_{0} y(x)+C_{1} h y^{\prime}(x)+\ldots C_{p} h^{p} y^{p}(x)+C_{p+1} h^{p+1} y^{p+1}(x)+C_{p+2} h^{p+2} y^{p+2}(x)+\ldots
$$

Theorem 1: The linear operator and the associated block method are said to be of order $p$ if $C_{0}=C_{1}=\ldots C_{p}=C_{p+1}=0, C_{p+2}=0, C_{p+2}=0, C_{p+3} \neq 0 \quad C_{p+3}$ is called the error constant. It implies that the local truncation error is given by

$$
T_{n+k}=C_{p+4} h^{p+3} y^{p+3}(x)+O\left(h^{p+4}\right)
$$

Expanding the block in Taylor Series gives

$$
\left.\left[\begin{array}{l}
\sum_{q=0}^{\infty} \frac{\left(\frac{1}{4} h\right)}{q !} y_{n}^{q}-y_{n}-\frac{1}{4} h y_{n}^{\prime}-\frac{1}{32} h^{2} y_{n}^{\prime \prime}-\frac{3929}{2580480} h^{3} y_{n}^{\prime \prime \prime}-\sum_{q=0}^{\infty} \frac{h^{q+3}}{q !}\left(\frac{995}{516096}\left(\frac{1}{4}\right)^{q}-\frac{1931}{1290240}\left(\frac{1}{2}\right)^{q}+\frac{173}{184320}\left(\frac{3}{4}\right)^{q}-\frac{1539}{286720}(1)^{q}+\frac{139}{2580480}\left(\frac{5}{4}\right)^{q}\right) \\
\sum_{q=0}^{\infty} \frac{\left(\frac{1}{2} h\right)}{q !} y_{n}^{q}-y_{n}-\frac{1}{2} h y_{n}^{\prime}-\frac{1}{8} h^{2} y_{n}^{\prime \prime}-\frac{317}{40320} h^{3} y_{n}^{\prime \prime \prime}-\sum_{q=0}^{\infty} \frac{h^{q+3}}{q !}\left(\frac{367}{20160}\left(\frac{1}{4}\right)^{q}-\frac{19}{2016}\left(\frac{1}{2}\right)^{q}+\frac{61}{10080}\left(\frac{3}{4}\right)^{q}-\frac{89}{40320}(1)^{q}+\frac{1}{2880}\left(\frac{5}{4}\right)^{q}\right) \\
\sum_{q=0}^{\infty} \frac{\left(\frac{3}{4} h\right)}{q !} y_{n}^{q}-y_{n}-\frac{3}{4} h y_{n}^{\prime}-\frac{9}{32} h^{2} y_{n}^{\prime \prime}-\frac{783}{40960} h^{3} y_{n}^{\prime \prime \prime}-\sum_{q=0}^{\infty} \frac{h^{q+3}}{q !}\left(\frac{16119}{286720}\left(\frac{1}{4}\right)^{q}-\frac{2187}{143360}\left(\frac{1}{2}\right)^{q}+\frac{423}{28672}\left(\frac{3}{4}\right)^{q}-\frac{1539}{286720}(1)^{q}+\frac{243}{286720}\left(\frac{5}{4}\right)^{q}\right) \\
\sum_{q=0}^{\infty} \frac{(1 h)}{q !} y_{n}^{q}-y_{n}-h y_{n}^{\prime}-\frac{1}{2} h^{2} y_{n}^{\prime \prime}-\frac{89}{2520} h^{3} y_{n}^{\prime \prime \prime}-\sum_{q=0}^{\infty} \frac{h^{q+3}}{q !}\left(\left(\frac{73}{630}\left(\frac{1}{4}\right)^{q}-\frac{1}{90}\left(\frac{1}{2}\right)^{q}+\frac{11}{315}\left(\frac{3}{4}\right)^{q}-\frac{5}{504}(1)^{q}+\frac{1}{630}\left(\frac{5}{4}\right)^{q}\right)\right) \\
\sum_{q=0}^{\infty} \frac{\left(\frac{5}{4} h\right)}{q !} y_{n}^{q}-y_{n}-\frac{5}{4} h y_{n}^{\prime}-\frac{25}{32} h^{2} y_{n}^{\prime \prime}-\frac{29125}{516096} h^{3} y_{n}^{\prime \prime \prime}-\sum_{q=0}^{\infty} \frac{h^{q+3}}{q !}\left(\left(\frac{101875}{516096}\left(\frac{1}{4}\right)^{q}-\frac{625}{258048}\left(\frac{1}{2}\right)^{q}+\frac{19375}{258048}\left(\frac{3}{4}\right)^{q}-\frac{625}{73728}(1)^{q}+\frac{1375}{516096}\left(\frac{5}{4}\right)^{q}\right)\right)
\end{array}\right]=\left[\begin{array}{l}
0 \\
0 \\
0 \\
0 \\
0
\end{array}\right]\right)
$$

and comparing the coefficients of $\mathrm{h}$, the order of the block is $p=6$ with error constant $C_{P+3}=\left[\frac{-82459}{7997698867200}, \frac{-4091}{62482022400}, \frac{-53487}{380842803200}, \frac{-13}{5857689600}, \frac{1700375}{959723864064}\right]$

\subsection{Consistency}

It is important that a linear multistep method satisfied the necessary and sufficient conditions. A numerical method is said to be consistent if the following conditions are satisfied.

i. The order of the scheme must be greater than or equal to 1 i.e. $\mathrm{p} \geq 1$.

ii. $\sum_{j=0}^{k} \alpha_{j}=0, \alpha_{j}$ 's are the coefficients of the first characteristic polynomial

iii. $\rho(r)=\rho^{\prime}(r)=0$ where $r=1$, root of the characteristics polynomial

$$
\begin{aligned}
\left|\left[\lambda A^{(0)}-A^{(i)}\right]\right| & =\left[\begin{array}{lllll}
1 & 0 & 0 & 0 & 0 \\
0 & 1 & 0 & 0 & 0 \\
0 & 0 & 1 & 0 & 0 \\
0 & 0 & 0 & 1 & 0 \\
0 & 0 & 0 & 0 & 1
\end{array}\right]-\left[\begin{array}{lllll}
0 & 0 & 0 & 0 & 1 \\
0 & 0 & 0 & 0 & 1 \\
0 & 0 & 0 & 0 & 1 \\
0 & 0 & 0 & 0 & 1 \\
0 & 0 & 0 & 0 & 1
\end{array}\right] \\
\lambda^{5}-\lambda^{4} & =0, \lambda=0,0,0,01
\end{aligned}
$$

the above conditions

\subsection{Region of Absolute Stability of the Method}

In (14), we express the stability in the form below [4, 13, $18]$. iv. $\rho^{(3)}(r)=3 ! \sigma(r)$ for $r=1, \sigma(\mathrm{r})$ is the second characteristic polynomial.

The first condition is a sufficient condition for the associated block method to be consistent [8, 9, 11-13].

\subsection{Zero Stability of the Method}

The general form of block method is given as

$$
A^{(0)} Y_{m}=A^{(i)} Y_{m-i}+h^{m u}\left[B^{i} Y_{m}+B^{i} Y_{m-i}\right]
$$

Applying (9-16) gives 
We express block method in the form

$$
\left[\frac{Y}{Y_{i+1}}\right]=\left[\begin{array}{ll}
A & U \\
B & V
\end{array}\right]\left[\frac{h^{2} f(y)}{Y_{i-1}}\right]
$$

$$
\begin{aligned}
& A=\left[\begin{array}{cccccc}
0 & 0 & 0 & 0 & 0 & 0 \\
\frac{3129}{2580480} & \frac{975}{516096} & \frac{-1931}{12910240} & \frac{173}{184320} & \frac{-1539}{286720} & \frac{139}{2580480} \\
\frac{317}{40320} & \frac{367}{20160} & \frac{-19}{2016} & \frac{61}{10080} & \frac{-89}{40320} & \frac{1}{2880} \\
\frac{783}{40960} & \frac{16119}{286720} & \frac{-2187}{143360} & \frac{423}{28672} & \frac{-1539}{286720} & \frac{243}{286720} \\
\frac{89}{2520} & \frac{73}{630} & \frac{-1}{90} & \frac{11}{315} & \frac{-5}{504} & \frac{1}{630} \\
\frac{-29125}{516096} & \frac{101875}{516096} & \frac{625}{258048} & \frac{19375}{258048} & \frac{-625}{73728} & \frac{1375}{516096}
\end{array}\right] \\
& B=\left[\begin{array}{cccccc}
\frac{3129}{2580480} & \frac{975}{516096} & \frac{-1931}{12910240} & \frac{173}{184320} & \frac{-1539}{286720} & \frac{139}{2580480} \\
\frac{-29125}{516096} & \frac{101875}{516096} & \frac{625}{258048} & \frac{625}{258048} & \frac{-625}{73728} & \frac{1375}{516096}
\end{array}\right], V=\left[\begin{array}{ll}
0 & 1 \\
0 & 1
\end{array}\right] \\
& U=\left[\begin{array}{ll}
0 & 1 \\
0 & 1 \\
0 & 1 \\
0 & 1 \\
0 & 1 \\
0 & 1
\end{array}\right], \quad M=\left[\begin{array}{lllll}
1 & 0 & 0 & 0 & 0 \\
0 & 1 & 0 & 0 & 0 \\
0 & 0 & 1 & 0 & 0 \\
0 & 0 & 0 & 1 & 0 \\
0 & 0 & 0 & 0 & 1
\end{array}\right], Y_{m}=\left[\begin{array}{c}
y_{n} \\
y_{n+\frac{1}{4}} \\
y_{n+\frac{1}{2}} \\
y_{n+\frac{3}{4}} \\
y_{n+1} \\
y_{n+\frac{5}{4}}
\end{array}\right], f(y)=\left[\begin{array}{c}
f_{n} \\
f_{n+\frac{1}{4}} \\
f_{n+\frac{1}{2}} \\
f_{n+\frac{3}{4}} \\
f_{n+1} \\
f_{n+\frac{5}{4}}
\end{array}\right], Y_{i-1}=\left[\begin{array}{l}
y_{n+1} \\
y_{n}
\end{array}\right], Y_{i+1}=\left[\begin{array}{l}
y_{n+1} \\
y_{n+\frac{5}{4}}
\end{array}\right]
\end{aligned}
$$

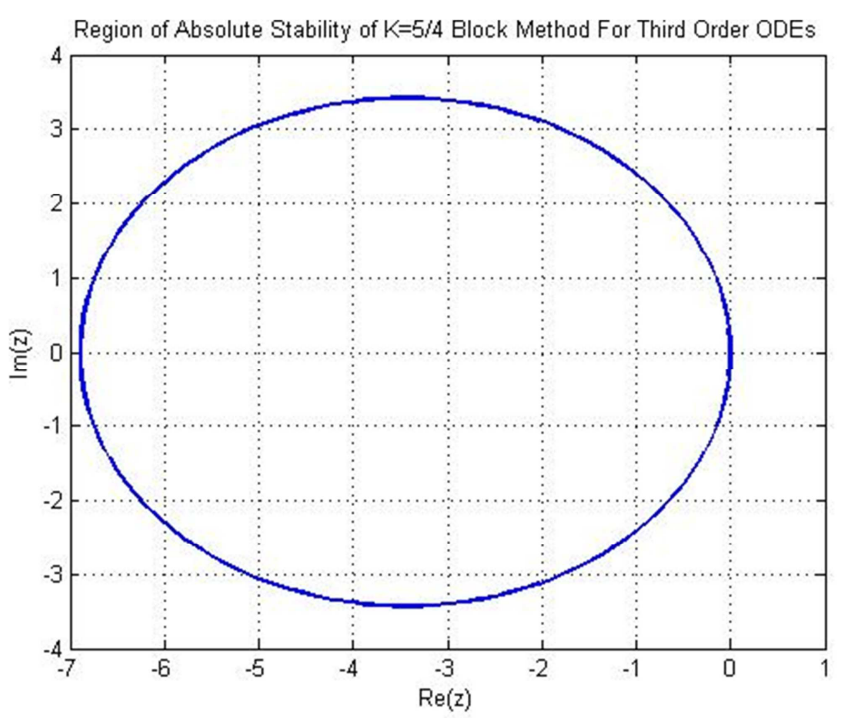

Figure 1. Region of Absolute Stability of the Method.

\section{Numerical Experiment}

In this section, the efficiency and the performance the new developed methods is tested on five test problems. We present some numerical experiments widely solved $[2,14$,
20]. The performance of the new developed method is examined on seven third-order initial value problems of ordinary differential equations. Tables 1-7 shows the comparison of our method with the selected problems in the existing methods $[2,14,20]$ in terms of absolute errors.

Problem 1

$$
y^{\prime \prime \prime}-y^{\prime \prime}+y^{\prime}-y=0, y(0)=1, y^{\prime}(0)=0, y^{\prime \prime}(0)=-1,0 \leq x \leq 1
$$

Exact soln: $y(x)=\cos x$

Problem 2

$$
\begin{gathered}
y^{\prime \prime \prime}+5 y^{\prime \prime}+7 y^{\prime}+3 y=0, y(0)=1, y^{\prime}(0)=0, y^{\prime \prime}(0)=-1, \\
-1,0 \leq x \leq 1
\end{gathered}
$$

Exact soln: $y(x)=e^{-x}+x e^{-x}$

Problem 3

$$
y^{\prime \prime}=3 \sin x, y(0)=1, y^{\prime}(0)=0, y^{\prime \prime}(0)=-2,0 \leq x \leq 1
$$

Exact soln: $y(x)=3 \cos x+\left(x^{2} / 2\right)-2$

Problem 4 


$$
\begin{aligned}
& y^{\prime \prime}=y^{\prime}\left(2 x y^{\prime \prime}+y^{\prime}\right) \\
& y(0)=1, y^{\prime}(0)=\frac{1}{2}, y^{\prime \prime}(0)=0, h=0.01
\end{aligned}
$$

Exact solution: $y(x)=1+\frac{1}{2} \operatorname{inl}\left(\frac{2+x}{2-x}\right)$

Problem 5

$$
\begin{aligned}
& y^{\prime \prime \prime}=x-4 y \\
& y(0)=0, y^{\prime}(0)=0, y^{\prime \prime}(0)=1, h=0.01
\end{aligned}
$$

Exact solution: $y(x)=\frac{-3}{16} \cos (2 x)+\frac{3}{16}+\frac{x^{2}}{8}$

Problem 6

The non-linear boundary layer flow problem

$$
2 y^{\prime \prime \prime}+y y^{\prime \prime}=0, y(0)=0, y^{\prime}(0)=0, y^{\prime \prime}(0)=1, \mathrm{~h}=0.1
$$

\begin{tabular}{|c|c|c|c|c|}
\hline $\mathbf{X}$ & Exact solution & Computed solution & Error in Proposed Method & Error in [14] \\
\hline 0.0100000 & 0.999950000417 & 0.999950000417 & $0.0000 \mathrm{e}+00$ & $6.72000 \mathrm{e}-07$ \\
\hline 0.0200000 & 0.999800006667 & 0.999800006667 & $1.1102 \mathrm{e}-16$ & $1.34410 \mathrm{e}-06$ \\
\hline 0.0300000 & 0.999550033749 & 0.999550033749 & $4.4409 \mathrm{e}-16$ & $2.01700 \mathrm{e}-06$ \\
\hline 0.0400000 & 0.999200106661 & 0.999200106661 & $5.8842 \mathrm{e}-15$ & $2.68840 \mathrm{e}-06$ \\
\hline 0.0500000 & 0.998750260395 & 0.998750260395 & $2.6201 \mathrm{e}-14$ & $3.35940 \mathrm{e}-06$ \\
\hline 0.0600000 & 0.998200539935 & 0.998200539935 & $8.3822 \mathrm{e}-14$ & \\
\hline 0.0700000 & 0.997551000253 & 0.997551000253 & $2.0750 \mathrm{e}-13$ & \\
\hline 0.0900000 & 0.995952733012 & 0.995952733013 & $8.4743 e-13$ & \\
\hline 0.1000000 & 0.995004165278 & 0.995004165280 & $1.5086 \mathrm{e}-12$ & \\
\hline
\end{tabular}

This is the famous Blasius equation

Problem 7

$$
y^{\prime \prime \prime}=-y^{\prime}, y(0)=0, y^{\prime}(0)=1, y^{\prime \prime}(0)=-2, h=0.1
$$

Table 1. Results of problem 1, $h=0.01$.

Table 2. Results of problem 2, $h=0.1 / 32$.

\begin{tabular}{llll}
\hline $\mathbf{X}$ & Exact solution & Computed solution & Error in our Method \\
\hline 0.0101563 & 0.999948773170 & 0.999948773170 & $7.9936 \mathrm{e}-15$ \\
0.0109375 & 0.999940619910 & 0.999940619910 & $1.1546 \mathrm{e}-14$ \\
0.0117188 & 0.999931869541 & 0.999931869541 & $1.5543 \mathrm{e}-14$ \\
0.0125000 & 0.999922523000 & 0.999922523000 & $2.2093 \mathrm{e}-14$ \\
0.0132813 & 0.999912581224 & 0.999912581224 & $3.0309 \mathrm{e}-14$ \\
0.0140625 & 0.999902045148 & 0.999902045148 & $4.0079 \mathrm{e}-14$ \\
0.0148437 & 0.999890915707 & 0.999890915707 & $5.1625 \mathrm{e}-14$ \\
0.0156250 & 0.999879193834 & 0.999879193834 & $6.4615 \mathrm{e}-14$ \\
0.0164063 & 0.999866880460 & 0.999866880460 & $8.2045 \mathrm{e}-14$ \\
0.0171875 & 0.999853976517 & 0.999853976517 & $1.0258 \mathrm{e}-13$ \\
\hline
\end{tabular}

Table 3. Results of problem $3, h=0.1$.

\begin{tabular}{llll}
\hline $\mathrm{X}$ & Exact solution & Computed solution & Error in our Method \\
\hline $\mathbf{0 . 1 0 0 0 0 0}$ & $\mathbf{0 . 9 9 0 0 1 2 4 9 5 8 3 4}$ & $\mathbf{0 . 9 9 0 0 1 2 4 9 5 8 3 4}$ & $\mathbf{2 . 2 2 0 4 e - 1 6}$ \\
\hline 0.200000 & 0.960199733524 & 0.960199733524 & $4.4409 \mathrm{e}-16$ \\
0.300000 & 0.911009467377 & 0.911009467377 & $1.3323 \mathrm{e}-015$ \\
0.400000 & 0.843182982009 & 0.843182982009 & $3.8858 \mathrm{e}-015$ \\
0.500000 & 0.757747685671 & 0.757747685671 & $9.2149 \mathrm{e}-015$ \\
0.600000 & 0.656006844729 & 0.656006844729 & $1.8985 \mathrm{e}-014$ \\
0.700000 & 0.539526561853 & 0.539526561853 & $3.4084 \mathrm{e}-014$ \\
0.800000 & 0.410120128041 & 0.410120128041 & $5.7343 \mathrm{e}-014$ \\
0.900000 & 0.269829904812 & 0.269829904812 & $9.0095 \mathrm{e}-014$ \\
1.000000 & 0.120906917604 & 0.120906917604 & 1.014 \\
\hline
\end{tabular}

Table 4. Results of problem $4, h=0.01$.

\begin{tabular}{llll}
\hline $\mathbf{X}$ & Exact solution & Computed solution & Error in our Method \\
\hline 0.0100000 & 1.005000041667 & 1.005000041667 & $2.2204 \mathrm{e}-16$ \\
0.0200000 & 1.010000333353 & 1.010000333353 & $0.0000 \mathrm{e}+00$ \\
0.0300000 & 1.015001125152 & 1.015001125152 & $1.9984 \mathrm{e}-15$ \\
0.0400000 & 1.020002667307 & 1.020002667307 & $1.4211 \mathrm{e}-14$ \\
0.0500000 & 1.025005210287 & 1.025005210287 & $5.5511 \mathrm{e}-14$ \\
0.0600000 & 1.030009004863 & 1.030009004863 & $1.6409 \mathrm{e}-13$ \\
0.0700000 & 1.035014302180 & 1.035014302180 & $3.8813 \mathrm{e}-13$ \\
0.0800000 & 1.040021353837 & 1.040021353836 & $7.9980 \mathrm{e}-13$ \\
0.0900000 & 1.045030411959 & 1.045030411958 & $1.5010 \mathrm{e}-12$ \\
0.1000000 & 1.050041729278 & 1.050041729276 & $2.6241 \mathrm{e}-12$ \\
\hline
\end{tabular}


Table 5. Results of problem $5, h=0.01$.

\begin{tabular}{|c|c|c|c|}
\hline $\mathbf{X}$ & Exact solution & Computed solution & Error in our Method \\
\hline 0.0100000 & 0.000049998750 & 0.000049998750 & $1.7896 \mathrm{e}-17$ \\
\hline 0.0200000 & 0.000199980001 & 0.000199980000 & $5.7085 \mathrm{e}-13$ \\
\hline 0.0300000 & 0.000449898762 & 0.000449898759 & $2.7015 \mathrm{e}-12$ \\
\hline 0.0500000 & 0.001249219010 & 0.001249218995 & $1.5592 \mathrm{e}-11$ \\
\hline 0.0600000 & 0.001798380777 & 0.001798380749 & $2.8358 \mathrm{e}-11$ \\
\hline 0.0700000 & 0.002447000710 & 0.002447000664 & $4.6545 \mathrm{e}-11$ \\
\hline 0.0900000 & 0.004041807602 & 0.004041807499 & $1.0308 \mathrm{e}-10$ \\
\hline 0.1000000 & 0.004987516655 & 0.004987516511 & $1.4336 \mathrm{e}-10$ \\
\hline
\end{tabular}

Table 6. Results of problem $5, h=0.1$.

\begin{tabular}{lllll}
\hline $\mathbf{X}$ & Exact solution & Computed solution & Error in our Method $(\mathbf{p}=\mathbf{6})$ & Error in $[\mathbf{2 0}](\mathbf{p}=\mathbf{7})$ \\
\hline 0.100000 & 0.004987516655 & 0.004987516655 & $2.1530 \mathrm{e}-013$ & $1.1899 \mathrm{e}-11$ \\
0.200000 & 0.019801063624 & 0.019801063633 & $8.5054 \mathrm{e}-012$ & $3.0422 \mathrm{e}-09$ \\
0.300000 & 0.043999572204 & 0.043999572273 & $6.8439 \mathrm{e}-011$ & $7.7796 \mathrm{e}-08$ \\
0.400000 & 0.076867491997 & 0.076867492292 & $2.9415 \mathrm{e}-010$ & $1.5559 \mathrm{e}-07$ \\
0.500000 & 0.117443317650 & 0.117443318550 & $8.9993 \mathrm{e}-010$ & $3.0541 \mathrm{e}-07$ \\
0.600000 & 0.164557921036 & 0.164557923257 & $2.2216 \mathrm{e}-009$ & $4.6102 \mathrm{e}-07$ \\
0.700000 & 0.216881160706 & 0.216881165434 & $4.7276 \mathrm{e}-009$ & $3.1380 \mathrm{e}-07$ \\
0.800000 & 0.003194884367 & 0.003194884368 & $7.4418 \mathrm{e}-013$ & $7.0374 \mathrm{e}-07$ \\
0.900000 & 0.004041807602 & 0.004041807603 & $1.1100 \mathrm{e}-012$ & $1.0177 \mathrm{e}-06$ \\
1.000000 & 0.004987516655 & 0.004987516656 & $1.5931 \mathrm{e}-012$ & $9.8800 \mathrm{e}-07$ \\
\hline
\end{tabular}

Table 7. Results of problem $6, h=0.1$.

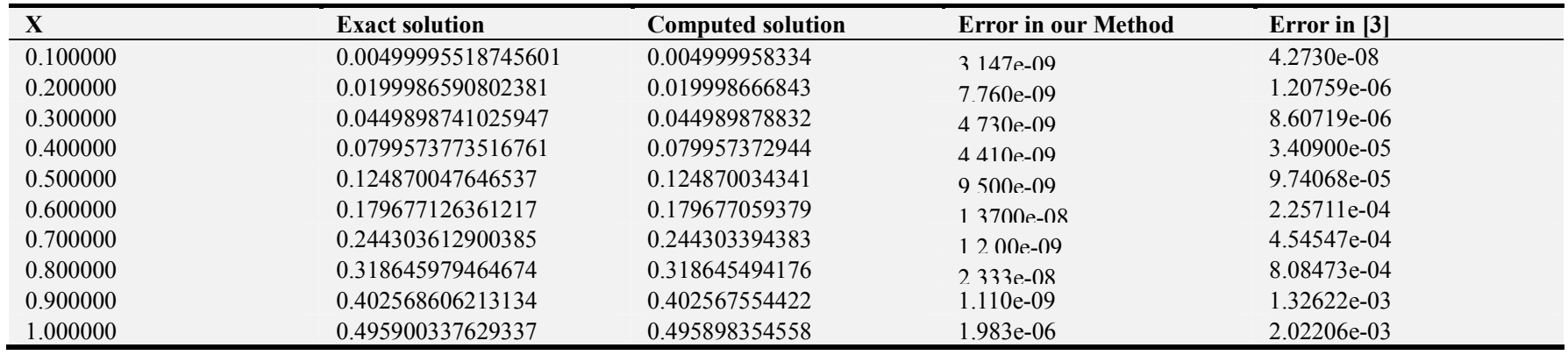

Table 8. Results of problem $7, h=0.1$.

\begin{tabular}{|c|c|c|c|c|c|}
\hline $\mathrm{X}$-value & Exact solution & Computed solution & Error in our Method & Error in [21] & Error in [22] \\
\hline 0.100000 & 0.089841747203 & 0.089841747203 & $3.3307 \mathrm{e}-016$ & 1.6088D-09 & $1.5405 \mathrm{e}-09$ \\
\hline 0.200000 & 0.158802486478 & 0.158802486477 & $3.6746 \mathrm{e}-013$ & 10387D-08 & $9.8455 \mathrm{e}-09$ \\
\hline 0.300000 & 0.206193184913 & 0.206193187532 & $2.6199 \mathrm{e}-009$ & 2.9572D-08 & $2.3652 \mathrm{e}-08$ \\
\hline 0.400000 & 0.231540330314 & 0.231540347621 & $1.7307 \mathrm{e}-008$ & $2.3147 \mathrm{D}-07$ & $4.3273 \mathrm{e}-08$ \\
\hline 0.500000 & 0.234590662385 & 0.234590720512 & $5.8127 \mathrm{e}-008$ & 4.5420D-07 & $3.9018 \mathrm{e}-08$ \\
\hline 0.600000 & 0.215313703214 & 0.215313845357 & $1.4214 \mathrm{e}-007$ & $1.4746 \mathrm{D}-06$ & $6.9700 \mathrm{e}-08$ \\
\hline 0.700000 & 0.173902061807 & 0.173902345471 & $2.8366 \mathrm{e}-007$ & 2.8734D-06 & $5.2032 \mathrm{e}-08$ \\
\hline 0.900000 & 0.026546846169 & 0.026547641357 & $7.9519 \mathrm{e}-007$ & $6.9217 \mathrm{D}-06$ & $4.7483 \mathrm{e}^{-07}$ \\
\hline 1.000000 & -0.077924403456 & -0.077923221234 & $1.1822 \mathrm{e}-006$ & 9.5974D-06 & $1.0693 \mathrm{e}-07$ \\
\hline
\end{tabular}

\section{Conclusions}

The development and implementation of a Fifth-fourth Continuous block implicit hybrid Method for the Numerical Solution of General Third Order Initial Value Problems in Ordinary Differential Equations is study in this research. In forming the method, the method of collocation approach was adopted by introducing off-grid points both at interpolation and collocations. The analysis of the method was studied and it was found to be consistent, convergent and zero-stable, with the region of absolute stable within which the method is stable. We further computed the order, error constants and plotted the region of absolute stability within which the method is stable. The absolute errors arising from Problems 1 - 5 using the new developed methods. Two of the problems were compared with the existing method. The results shows that the results displayed in tables 1 and 3 that the newly derived method performs better than the existing method, which are of the same order of accuracies. 


\section{References}

[1] Fatunla, S. O. (1988): Numerical methods for initial value problems in ordinary differential equations, Academic press Inc. Harcourt Brace Jovanovich Publishers, New York.

[2] A. Olaide Adesanya, D. Mfon Udoh and, A. M. Ajileye (2013): A New Hybrid Block Method For The Solution Of General Third Order Initial Value Problems Of OrdinaryDifferential Equations: International Journal of Pure and Applied Mathematics Volume 86 No. 2, 365-375.

[3] Adoghe L. O, Ogunware B. G and Omole E. O (2016): A family of symmetric implicit higher order methods for the solution of third order initial value problems in ordinary differential equations: Journal of Theoretical Mathematics \& Applications, 6 (3): 67-84.

[4] Adoghe L. O and Omole E. O (2018): Comprehensive Analysis of 3-Quarter-Step Collocation Method for Direct Integration of Second Order Ordinary Differential Equations Using Taylor Series Function. ABACUS (Mathematics Science Series) Vol. 44, N0 2, pp. 311-321.

[5] Awoyemi D. O (2001): A new Sixth -Order Algorithm for General Second Order Ordinary Differential Equations. International Journal of Computer mathematics, Vol 77, pp. $117-124$.

[6] Awoyemi, D. O and Idowu, O. M. (2005): A class of hybrid collocation Method for third ordinary differential equations. International Journal of Computer Math, 82 (10), 1287-1293.

[7] Atkinson K. E (1989): An introduction to Numerical Analysis, $2^{\text {nd }}$ Edition, John Wiley and Sons, New York.

[8] Fatokun, J. O (2007): Continuous Approach for deriving Self -starting multistep methods for initial value problems in ordinary differential Equations. Journal of Engineering and Applied Sciences Vol. 2 (3) pp. 504-508.

[9] Jain. M. K, Iyengar, S. R. K, Jain, R. K (2008): Numerical Methods for scientific and engineering computations (fifth edition). New Age International Publishers Limited.

[10] Henrici, P. (1962): Discrete Variable method in ordinary differential equations, John Wiley and Sons, New York.

[11] Kayode, S. J and A. Adeyeye (2011): A 3-step hybrid method for direct solution of second order initial value problems, Aust. J. of Basic and Applied Sciences, 5, No. 12 (2011), 2121-2126.
[12] Lambert J. D (1973): Computational Methods in ODES. John Wiley \& Sons, New York.

[13] Lambert J. D. (1991): Numerical methods for initial value problems in ordinary differential equation, New York, Academics Press Inc.

[14] Mohammed U. and R. B Adeniyi (2014): A Three Step Implicit Hybrid Linear Multistep Method for the Solution of Third Order Ordinary Differential Equations. Gen. Math. Notes, Vol. 25, No. 1, pp. 62-74.

[15] Samuel. N. Jator (2008): On the numerical integration of third order boundary value problems by a linear multistep method; International Journal of Pure and Applied Mathematics, Vol. 46, No 3,375-388.

[16] Skwame, Y., Sabo, J. \& Kyagya, T. Y. (2017): The constructions of implicit one-step block hybrid methods with multiple off-grid points for the solution of stiff ODEs. JSRR, $16(1): 1-7$.

[17] Tumba, P., Sabo, J. \& Hamadina, M., (2018): Uniformly Order Eight Implicit Second Derivative Method for Solving Second- Order Stiff Ordinary Differential Equations ODEs. Academic Journal of Applied Mathematical Sciences, 4: 43-48.

[18] Ogunware B. G, Adoghe L. O, Awoyemi D. O, Olanegan O. O., and Omole E. O (2018): Numerical Treatment of General Third Order Ordinary Differential Equations Using Taylor Series as Predictor. Physical Science International Journal, 17 (3): 1-8; DOI: 10.9734/PSIJ/2018/22219.

[19] Yakubu D. G., Manjah N. H, Buba. S. S, and Masksha. A. I. (2011): A family of uniform accurate Lobatto-Runge-Kutta collocation methods: Journal of Computational and Applied Mathematics., Vol. 30, N. 2, pp 315-330.

[20] Awoyemi D. O., Kayode S. J. and Adoghe L. O (2014).: A four-point fully implicit method for numerical integration of third-order ordinary differential equations, Int. J. Physical Sciences, 9 (1), 7-12.

[21] T. A. Anake, A. O. Adesanya. G. J. Oghonyon, and M. C. Agarana (2013): Block Algorithm For General Third Order Ordinary Differential, ICASTOR Journal of Mathematical Sciences Vol. 7, No. 2, $127-136$.

[22] A. O. Adesanya, M. O. Udoh, A. M. Alkali (2012) A new block-predictor corrector algorithm for the solution of $y^{\prime \prime \prime}=f$ $\left(x, y, y^{\prime}, y^{\prime \prime}\right)$, American J. of Computational Mathematics, (2), 341-344. 